\title{
A Benchmarking application on Workload and Performance forecasting of micro services
}

\author{
R. Divya Mounika ${ }^{1}$, R .Naresh ${ }^{2 *}$ \\ ${ }^{1}$ M.Tech-Student, Department of Computer Science and Engineering, SRM Institute of Science and Technology, \\ Kattankulathur, Chengalpattu, Chennai, Tamilnadu, India-603 203. \\ ${ }^{2 *}$ Associate Professor, Department of Computer Science and Engineering, SRM Institute of Science and \\ Technology, Kattankulathur, Chengalpattu, Chennai, Tamilnadu, India-603 203. \\ ${ }^{1}$ rm2769@srmist.edu.in, ${ }^{2 *}$ nareshr@ srmist.edu.in
}

Article History: Received: 11 January 2021; Accepted: 27 February 2021; Published online: 5 April 2021

\begin{abstract}
Micro services are increasingly understood as the ideal architectural framework for building large cloud applications within and beyond organizational boundaries. These micro services architectures scale up the application, but are expensive to work on, so pay attention to workflow planning and workflow planning. However, this issue is not very clear. In this work, we are developing independent micro services workflows suitable for modeling and prediction methods and designing three-step game models for based applications. Solved the problem of designing micro services based applications to reduce end-to-end delays under userspecific limitations (MAWS-BC) and recommended micro services routing algorithms. The design process and estimation methods are improved and adequate. The experimental results produced by a well-known micro service bank cover a wide variety of statistical analyzes and the production utility of graphic design is shown by a large comparison copy compared to current algorithms.
\end{abstract}

Keywords: micro services,routing, Cloud computing, databases

\section{Introduction}

New micro service architectures are increasingly being incorporated into their application systems and development. Micro service applications running in the cloud typically involve the implementation and interaction of multiple micro services that can be pushed, pushed and updated without compromising performance [1-5]. This independence in micro services management increases, simplifies, updates, and creates applications, but also increases performance upwards and complicates the impact of useowners. Therefore, modeling and programming tasks are a major concern in designing cloud-based micro- service based applications unlike traditional non-traditional applications, applications used by micro services pose two new challenges in their workflow and programming work:i) Application of features, workloads for micro services behavior. I s. And ii) When calculating the public clouds a paid user, application providers have reduced costs in addition to achieving the traditional goal of improving services in a dynamic environment that many users share with long-term uncertainty [6-10]. These disruptive methods, conflicts, and conflicts can be summarized at the container level. Specifically special, containers can be filled [11-15]. There are certain limits on the CPU and memory resources consumed by the service model, which allows the service to be private and have access to other virtual resources.

\section{Literature survey}

\subsection{A Systematic Mapping Study}

Microservices have recently emerged as an architectural framework, discussing how to build, manage, and upgrade infrastructure for small private sectors. Especially in the cloud, the micro services system architecture appears to be a significant complement to PaaS-level box technology. However, no secondary school is currently promoting this research. This means identifying, distributing and comparing patents on micro services and their cloud research institutes [16]. We conducted a systematic review of 21 selected study maps published in the last two years from the emergence of a limited number of services to the end of 2015, and classified and compared selected studies on a case-by-case basis [17]. This leads to a debate about what is accepted and developed in micro services architectures, not only putting the micro services architecture in a continuous development environment, but also moving the technology closer to the cloud. Recently, a micro services architecture was introduced to distribute application architectures to self-contained implementation services, allowing them to quickly adapt to any infrastructure as needed [18-21]. Micro services can be pushed independently and are usually supported by support and music systems. For example, in the cloud, you can send microservices on a regular basis, sometimes on your own.Microservices and musical clutter between delivery and cloud environments are a component of the home. Sounds provides management tools for flexible music production and compatibility, especially when it comes to PaaS[22,23]. 


\subsection{Performance Debugging for Distributed Systems of Black Boxes}

Many attractively sized systems are spread across multiple networks. Such systems can be less likely to make mistakes, especially if they do not work properly. The problem is further complicated by the fact that the system consists of "black box" elements. Software from different vendors (and in some cases competitors), usually without source code. Employees who provide everyday solutions do not always have the skills and expertise to effectively destroy these systems. Our goal is to design tools that allow authors to focus their implementation process on a distributed system integrated with a black box enclosure. This issue is resolved by getting a signal level signal from the operating system. This allows you to implement it without knowing the contents of the node or the meaning of the message. Various algorithms have been developed to minimize the causal path through the distribution system for these signals. One of them is to use RPC to interact with the data and create a causal relationship between the calls. Someone is using signal processing technology. My proposed algorithm can recommend wasting time for other foods from other relationships.

\subsection{Using Performance Prediction to Allocate Grid Resources}

Major application usually requires a computer system to restore the computer's murder system for processing. Generally, users are able to collect resources that can be used to think. The resources can be strong, and users need to decide that is distributed the virus and natural resources to use. However, most of users need an automatic device to process the automatic adults, as many users have knowledge of all items used and they have information. This project is presenting the arrangements of the operating system. According to historical data, to find the correct factor. The mathematical test system is used, the first processing period is shown.

\subsection{Existing system}

The compression system strives to predict future performance using highly accurate data and multidimensional results, and calculates the target engine in terms of the value of the historical application generated by the tool. Predict the implementation process. The method of predicting the dynamic time. Using different rotation probes to obtain a standardized control system as a training, we obtained a complete estimate of the computational performance as the number of nodes increases, predicting the implementation time on larger devices.

\subsection{Disadvantages}

You can create and discuss drugs at the first challenge, boxes, and "system" systems, and drug levels. You can insert other restrictions from CPU memory and consumed memory every day and receive a series of services. As a result, there is no need to convince interference or problem issues. However, monitoring DRAMA and design frames collect measurements to use CPU balance, but cannot use networks. The main method is also performed and Strategy with a simple process.

\section{Proposed system}

Adding independent micro services and designing systems, three-step workflow design for micro services and small micro services applications to reduce end-to-end latency under specific user budgets. Schedule problems planning your application. Limitations in the public cloud called MAWS-BC recommend heuristic programming solutions for this problem for a single application that contains multiple micro services.

The proposed approackh follows a complex sequence of steps and differs from the approach in two ways:

i) Considersmall business theft, business management, and data processingprocesses.

ii) Asfar as weareconcerned, our first job will be to be able to model and predict the advantages of small cloud-based service applications.

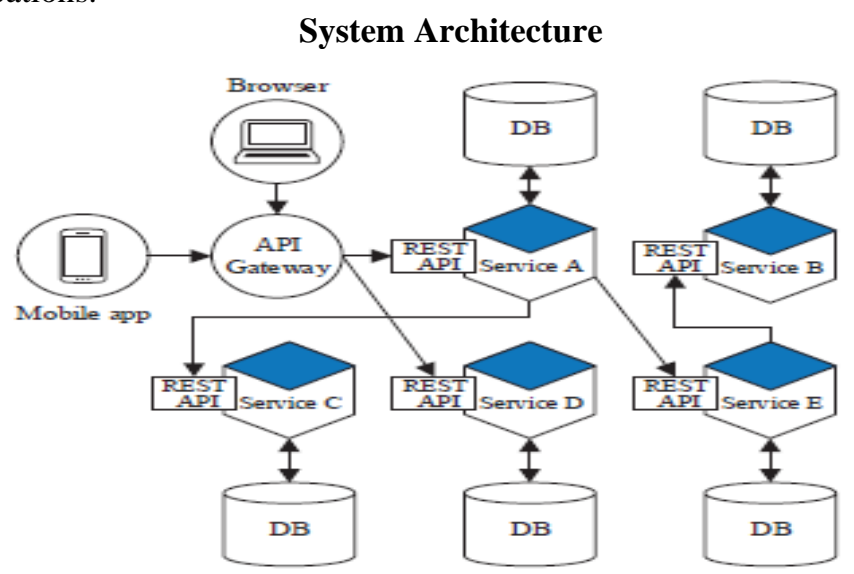

Fig . 1. Micro service architecture 
The micro services function translates the system that organizes applications that are easy to integrate, integrate, and integrate into a collection of services. Each service makes a set of appropriate connections. This service is developed and used independently in several locations and uses synchronization protocols such as: As an unmatched protocol such as HTTP / REST or AMQP for communication. Each service is usually linked to a database to separate it from other services.

\section{System Implementation \\ 4.1 Aggregator}

By supporting the number of people, application provisions can determine the required settings to pay some business needs and their relationship. So this application is published to the same installation issue called Hells. She is hiding an internal building and provides special apis for any user.

\subsection{Deployment:}

During the push-up operation, each small task is put in a shutdown mode called a box to provide specific CPU, memory, and I / O components. These containers are grouped and operate in a collection of similar devices interconnected (VMs) provided by the provider cloud. society.

\subsection{Execution phase:}

Many users stop related to news and, therefore, send requests only with specific budget limits.

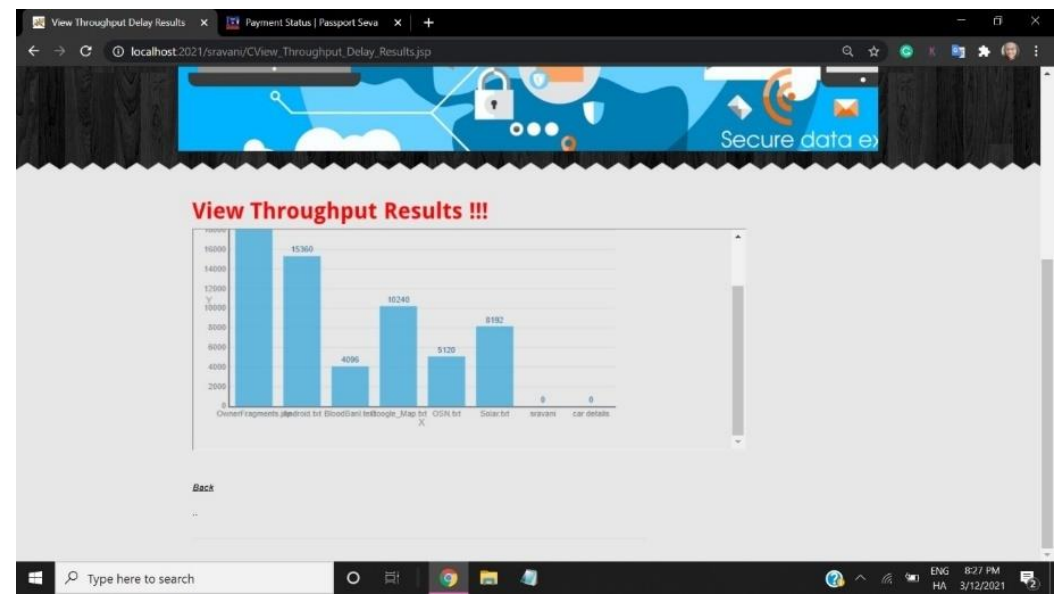

Fig. 2. Comparison of various protocols using throughput parameter.

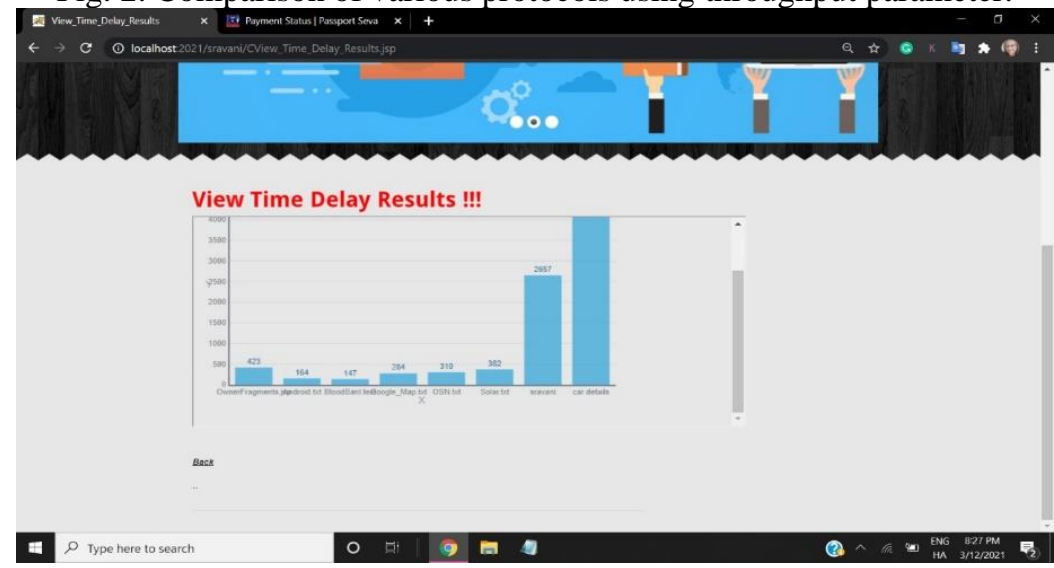

Fig. 3. Computation time for various protocols.

\section{Conclusion}

Small business modeling is gaining acceptance in schools and the industry. Workloads and workloads are two of the most important topics for small business applications. In this regard, we develop application performance modeling and forecasting methods. Small service. Provides solutions to MAWS-BC issues and work issues. My proposed work has improved performance and optimization through test and simulation results, and has great potential to improve the performance of many short-lived service applications. Set up in the public cloud. 


\section{Future scope}

First, the MAWS-BC issue under investigation focuses on establishing applications only for microorganisms. We plan to consider assigning services to micro services and deploying micro services to modern applications at the same time, resulting in a complex two-stage upgrade.

\section{References}

1. M. Fowler and J. Lewis, "Microservices," ThoughtWorks. http://martinfowler.com/articles/microservices.html [last accessed on February 11, 2017], 2014.

2. T. Ueda, T. Nakaike, and M. Ohara, "Workload characterization for microservices," in Workload Characterization (IISWC),IEEE, 2016, pp. 1-10.

3. M. Fazio, A. Celesti, R. Ranjan, C. Liu, L. Chen, and M. Villari, "Open issues in scheduling microservices in the cloud," IEEE Cloud Computing, vol. 3, no. 5, pp. 81-88, 2016.

4. C. Pahl and P. Jamshidi, "Microservices: A systematic mapping study," in Proceedings of the 6th International Conference on Cloud Computing and Services Science, 2016, pp. 137-146.

5. Pattern: Service instance per container: https://microservices.io/patterns/deployment/service-percontainer.html.

6. Nagios, "Nagios it infrastructure monitoring," https://www.nagios.org/ [last accessed on February 11, 2017], 2017.

7. Ganglia, "Ganglia monitoring system," http://ganglia.info/, 2017.

8. Heapster, "Compute resource usage analysis and monitoring of container clusters," https://github.com/kubernetes/heapster [last accessed on February 11, 2017], 2017.

9. Kubernetes, "Compute resource usage analysis and monitoring of container clusters," https://kubernetes.io/, 2017.

10. Mesos, “Apache mesos," http://mesos.apache.org/, 2017.

11. C.N.S.Vinoth Kumar, A.Suhasini, "Secured Three-Tier Architecture for Wireless Sensor Networks Using Chaotic Neural Networks", 'Advances in Intelligent Systems and Computing' AISC Series, Springer Science + Business Media Singapore 2017 Vol. No. 507, Chapter No. 13, pp. No. 129-136,

12. GautamSrivastava, C.N.S. Vinoth Kumar, V Kavitha, N Parthiban, RevathiVenkataraman, "Two-Stage Data Encryption using Chaotic Neural Networks", Journal of Intelligent and Fuzzy systems, Vol. no.38, Issue. No.3, pp no.2561-2568, March 2020.

13. PraharshaSarma, Utkarsh Kumar, C.N.S.Vinoth Kumar, M.VasimBabu, "Accident Detection And Prevention Using Iot\& Python Opencv", International Journal Of Scientific \& Technology Research(IJSTR), Volume 9, Issue 04,pp no. 2677-2681, ISSN No: 2277-8616 April 2020.

14. M.VasimBabu, C.N.S. Vinoth Kumar, M.Venu, International journal entitled "Improvisation of localization accuracy using ERSSI based on ADV-HOP algorithm in wireless sensor network", International journal of innovative technology and exploring engineering (IJITEE), Feb 2019

15. A.Saranya, R.Naresh "Cloud Based Efficient Authentication for Mobile Payments using Key Distribution Method", Journal of Ambient Intelligence and Humanized Computing, Springer, 02 January, 2021. https://link.springer.com/article/10.1007\%2Fs12652-020-02765-7

16. R.Naresh, P.Vijayakumar, L. Jegatha Deborah, R. Sivakumar, “A Novel Trust Model for Secure Group Communication in Distributed Computing", Special Issue for Security and Privacy in Cloud Computing, Journal of Organizational and End User Computing, IGI Global, Vol.32, No. 3, Septemer 2020, Pp. 1-14.

17. R.Naresh, M.Sayeekumar, G.M.Karthick, P.Supraja, "Attribute-based hierarchical file encryption for efficient retrieval of files by DV index tree from cloud using crossover genetic algorithm",Soft Computing, Springer, Vol.23, No. 8, 2019, Pp. 2561-2574.

18. R Divya Mounika, R.Naresh, "The concept of Privacy and Standardization of Microservice Architectures in cloud computing”, European Journal of Molecular \& Clinical Medicine, Vol 7, No 2, Pages 5349-5370, Dec 2020.

19. P.Vijayakumar, R.Naresh, L. Jegatha Deborah, SK Hafizul Islam, “An efficient group key agreement protocol for secure P2P communication", Security and Communication Networks, Wiley, Vol.9, No.17, pp.3952-3965, 2016

20. P.Vijayakumar, R.Naresh, SK Hafizul Islam, L. Jegatha Deborah “An Effective Key Distribution for Secure Internet Pay-TV using Access Key Hierarchies”, Security and Communication Networks, Wiley, Vol.9, No.18, pp.5085-5097, 2016.

21. R. Naresh, M Meenakshi, G Niranjana, "Efficient study of Smart Garbage Collection for Ecofriendly Environment", Journal of Green Engineering, Vol.10, No.1, pp.1-10,Feb 2020.

22. Padmashree A., Prasath N. "Enabling Data Storage on Fog-An Attempt Towards IoT" In: Smys S., Bestak R., Chen JZ., Kotuliak I. (eds) International Conference on Computer Networks and Communication Technologies. Lecture Notes on Data Engineering and Communications Technologies, vol 15, 2019. Springer, Singapore. https://doi.org/10.1007/978-981-10-8681-6_82 
23. R. Jeevitha and N. Prasath, "Recrimination to Accident Using IoT Components for Healthcare Applications-A SURVEY," 2018 International Conference on Soft-computing and Network Security (ICSNS), Coimbatore, 2018, pp. 1-6, doi: 10.1109/ICSNS.2018.8573633. 